\title{
Diffuse intrinsic pontine glioma cells are vulnerable to low intensity electric fields delivered by intratumoral modulation therapy
}

\author{
Andrew Deweyert ${ }^{1} \cdot$ Erin Iredale ${ }^{2} \cdot \mathrm{Hu} \mathrm{Xu}^{3} \cdot$ Eugene Wong $^{2} \cdot$ Susanne Schmid ${ }^{1} \cdot$ Matthew O. Hebb $^{1,3}$ (1)
}

Received: 29 January 2019 / Accepted: 4 March 2019 / Published online: 9 March 2019

(c) The Author(s) 2019

\begin{abstract}
Introduction Diffuse intrinsic pontine glioma (DIPG) is a high fatality pediatric brain cancer without effective treatment. The field of electrotherapeutics offers new potential for other forms of glioma but the efficacy of this strategy has not been reported for DIPG. This pilot study evaluated the susceptibility of patient-derived DIPG cells to low intensity electric fields delivered using a developing technology called intratumoral modulation therapy (IMT).

Methods DIPG cells from autopsy specimens were treated with a custom-designed, in vitro IMT system. Computer-generated electric field simulation was performed to quantify IMT amplitude and distribution using continuous, low intensity, intermediate frequency stimulation parameters. Treatment groups included sham, IMT, temozolomide (TMZ) chemotherapy and radiation therapy (RT). The impact of single and multi-modality therapy was compared using spectrophotometric and flow cytometry viability analyses.

Results DIPG cells exhibited robust, consistent susceptibility to IMT fields that significantly reduced cell viability compared to untreated control levels. The ratio of viable:non-viable DIPG cells transformed from $\sim 6: 1$ in sham-treated to $\sim 1.5: 1$ in IMT-treated conditions. The impact of IMT was similar to that of dual modality TMZ-RT therapy and the addition of IMT to this treatment combination dramatically reduced DIPG cell viability to $\sim 20 \%$ of control values.

Conclusions This proof-of-concept study provides a novel demonstration of marked DIPG cell susceptibility to low intensity electric fields delivered using IMT. The potent impact as a monotherapy and when integrated into multi-modality treatment platforms justifies further investigations into the potential of IMT as a critically needed biomedical innovation for DIPG.
\end{abstract}

Keywords Electrotherapy · Glioma · Pediatric $\cdot$ Brain cancer · Electric fields · H3 K27 mutant

\section{Introduction}

Diffuse intrinsic pontine glioma (DIPG), also called H3 K27M-mutant diffuse midline glioma, is the most common brainstem cancer in children, representing $80 \%$ of tumors in this region of the central nervous system (CNS)

Matthew O. Hebb

mhebb@uwo.ca

1 Department of Anatomy and Cell Biology, Schulich School of Medicine and Dentistry, Western University, London, ON, Canada

2 Department of Medical Biophysics, Schulich School of Medicine and Dentistry, Western University, London, ON, Canada

3 Department of Clinical Neurological Sciences, Schulich School of Medicine and Dentistry, Western University, London, ON, Canada
$[1,2]$. The average age at diagnosis is $6-9$ years old and affected children typically present with a progressive spectrum of neurological deficits. Magnetic resonance imaging most often reveals an expansile, T2-hyperintense, poorlyenhancing mass centered in the pons [3]. The infiltration of DIPG within the brainstem parenchyma precludes safe surgical resection. The mainstay of treatment is fractionated radiation therapy (RT) which can provide transient symptom and tumor control resulting in median patient survival of 9 months and a 2-year survival rate $<10 \%$ [4]. Multiple chemotherapy protocols and agents have been trialed but have not demonstrated further survival benefit $[5,6]$. Despite international efforts in defining the molecular underpinnings of DIPG, there have been no recent therapeutic advances that substantially improve patient outcomes [2,4].

There is emerging evidence that electrotherapy may offer a novel means to control malignant glioma and considerable progress has been made treating the high 
fatality CNS cancer, glioblastoma (GBM). A single clinical electrotherapy system with demonstrated survival benefit has been approved for new and recurrent GBM. Unfortunately this device is not engineered for the treatment of infratentorial tumors such as DIPG [7-9]. Current PubMed searches using the terms diffuse intrinsic pontine glioma and electrotherapy or electric fields produced no reports. Thus, the potential impact of electrotherapy on DIPG remains unknown. Intratumoral modulation therapy (IMT) is a developing technology which may offer an electrotherapeutic option for tumors located anywhere within the CNS, including the brainstem [10, 11]. IMT exploits the electrosensitivity of cancer cells using implanted fieldgenerating sources (e.g., bioelectrodes) to deliver nonablative, low intensity electric fields that attenuate tumor growth and bolster multi-modality treatment platforms. Preclinical studies demonstrated potent efficacy of IMT as a monotherapy and when combined with chemotherapeutic or oncogene-silencing agents in primary human GBM cells and in vivo allogeneic GBM models $[10,11]$. To date, however, there have been no reports of the application of IMT to other CNS cancers. The goal of this pilot study was to determine the vulnerability of patient-derived DIPG cells to low intensity electric fields delivered using an established IMT protocol. The impact of IMT on DIPG resistance to conventional radiation and chemotherapy options was also investigated.

\section{Materials and methods}

\section{Patient-derived DIPG cells}

Patient-derived DIPG cells, labeled SU-DIPG-IV, SUDIPG-XIX and SU-DIPG-XXIV, were kindly received from Dr. Michelle Monje at Stanford University. The tumor collection protocol, culture methods at derivation, patient demographics and tumor genetics have been previously described [12-14]. The cells were derived from early postmortem DIPG specimens in three pediatric patients, aged 2, 2 and 6 years old respectively, who had received radiation and chemotherapy during their care. Upon arrival to the Hebb lab, frozen DIPG cells were thawed and cultured in 6-well plates (Corning, NY, United States) at $37^{\circ} \mathrm{C}$ with humidified air containing $5 \% \mathrm{CO}_{2}$ using NeuroCult ${ }^{\mathrm{TM}} \mathrm{NS}-\mathrm{A}$ medium supplemented with $10 \%$ proliferation supplement, bFGF (10 ng/mL), EGF (20 ng/mL), heparin sulfate $(2 \mu \mathrm{g} /$ $\mathrm{ml}$ ) and $1 \%$ penicillin/streptomycin (Complete NSA; Stemcell Technologies, Vancouver, B.C., Canada). The medium was changed every $72 \mathrm{~h}$ and cultures passaged 1:2 at 70-90\% confluence.

\section{In vitro IMT model}

The impact of IMT alone and combined with temozolomide (TMZ) chemotherapy and/or RT was evaluated using primary human DIPG cells cultured in $35 \mathrm{~mm}$ wells. The IMT model was created by fitting each well with a clinical grade, platinum-based strip bioelectrode (Ad-Tech Medical Instrument Corporation, Oak Creek, WI, USA) around the periphery and platinum-iridium bioelectrode (Medtronic Ltd., Hertfordshire, United Kingdom) at the center of the well and cell culture $[10,11]$. A waveform generator (Rigol DG1022; Electro-Meters Ltd., Pickering, ON, Canada) was used to deliver biphasic sinusoidal pulses with low amplitude ( $\pm 2 \mathrm{~V}$; peak-to-peak $4 \mathrm{~V}$ ) and intermediate frequency $(200 \mathrm{kHz})$ continuously over 3 days. Control wells (i.e. sham-treated) were fitted with bioelectrodes but did not receive stimulation.

\section{IMT field simulation}

The IMT electric field was simulated with COMSOL Multiphysics v 5.2a (Comsol Inc., Burlington, MA, USA) AC/ DC module, electric currents user interface in the frequency domain. The geometries of the in vitro model were manually created utilizing COMSOL Geometry to generate an identical in silico model. The dish and electrode apparatus were mimicked in geometry and the appropriate materials simulated utilizing known material conductance and dielectric properties. The boundary conditions were applied as the edge of the $35 \mathrm{~mm}$ dish. The centre stimulating electrode was set to emit a $2 \mathrm{~V}$ amplitude waveform, and the outer electrodes were grounds. Electrical insulation on outer boundaries was assumed. A mesh of the geometry was created, and a frequency of $200 \mathrm{kHz}$ chosen for evaluation. The dimensions and amplitude of the electric field within the culture dish were analyzed using MATLAB R2015b (MathWorks Inc., Natick, MA, United States).

\section{Multi-modality treatment of DIPG cells}

DIPG cells $\left(1 \times 10^{5}\right)$ treated with $50 \mu \mathrm{m} \mathrm{TMZ} \mathrm{were} \mathrm{cultured}$ in $35 \mathrm{~mm}$ wells fitted with IMT bioelectrodes without stimulation (i.e., sham IMT) for TMZ monotherapy or while receiving concurrent IMT during dual therapy. This TMZ concentration corresponds to plasma levels obtained with $150 \mathrm{mg} / \mathrm{m}^{2}$ in the adjuvant treatment phase of other forms of glioma [15]. Additional DIPG samples received 4 Gy of ionizing radiation in a single fraction using a Cobalt-60 irradiator with average dose rate of $74 \mathrm{cGy} / \mathrm{min}$. This dose was chosen after a RT dose-response study to permit evaluation of cooperative benefits between different therapeutic modalities 
in vitro $[16,17]$. The cells were allowed to recover for $1 \mathrm{~h}$ after RT and were then plated into $35 \mathrm{~mm}$ wells fitted with the IMT system. At this point, DIPG cells were cultured with no further treatment (i.e., RT monotherapy group), or in the presence of TMZ $(50 \mu \mathrm{M})$ or IMT (i.e., TMZ-RT or IMT-RT dual therapy groups), or both TMZ and IMT (i.e., TMZ-RTIMT multi-modality therapy group) for 3 days.

\section{Cell viability assay}

Cell viability was evaluated using the 3-(4,5-dimethylthiazol-2-yl)-2,5-diphenyltetrazolium (MTT) spectral analysis (Sigma Aldrich, St. Louis, MO, United States). This colorimetric assay measures the reduction of yellow MTT by mitochondrial succinate dehydrogenase to an insoluble, dark purple formazan product. Immediately following the DIPG cell treatments described above, MTT $(80 \mu \mathrm{l}$ at $5 \mathrm{mg} / \mathrm{ml})$ was added to the $35 \mathrm{~mm}$ wells and incubated for $3 \mathrm{~h}$ at $37^{\circ} \mathrm{C}$ in a humidified $5 \% \mathrm{CO}_{2}$ atmosphere. The cells were then lysed to release the purple formazan product by the addition of $300 \mu$ dimethyl sulfoxide for $15 \mathrm{~min}$ at room temperature. Absorbance was measured using an Epoch microplate spectrophotometer (BioTek, Winooski, VT, United States). Cell viability was estimated using optical density values at $570 \mathrm{~nm}$ with references at $655 \mathrm{~nm}$ detected in each well. Brightfield images of cells stained with MTT were obtained using a Motic AE31 inverted microscope fitted with an Infinity 1-3 scientific complementary metal-oxide semiconductor camera (Lumenera Corp, Nepean, ON, Canada).

\section{Flow cytometry}

Annexin V apoptosis detection with zombie red (ZR) was used to quantify fractions of live, apoptotic and dead DIPG cells, as per the manufacturer's instructions (BioLegend, San Diego, CA, USA). Cell fractions were analyzed using a Becton Dickinson LSR II SORP flow cytometer running FACSDiva software (BD Biosciences, Mississauga, ON, Canada). Cells were first gated on forward scatter (FSC-) versus side scatter (SSC-) characteristics before excluding doublets using consecutive gating FSC-Area versus FSC-Width and SSC-Area versus SSC-Width plots. The populations of annexin $\mathrm{V}+/ \mathrm{ZR}-$, annexin $\mathrm{V}+/ \mathrm{ZR}+$, annexin $\mathrm{V}-/ \mathrm{ZR}+$ and annexin $\mathrm{V}-\mathrm{ZZR}-$ were then calculated with quadrant gates. Approximately 25,000 single cells were acquired per sample at a maximum event rate of 5000 events per second. Data were analyzed using FlowJo v 9.6.3 software (TreeStar Inc., Ashland, OR, USA).

\section{Statistical analysis}

A $t$ test was used to compare paired data sets. Multiple pairwise comparisons were performed using one-way analysis of variance (ANOVA) followed by Tukey post hoc analysis (SPSS Inc., Chicago, IL, USA). Data are presented as mean \pm standard deviation with significance assumed at $\mathrm{p}<0.05$.

\section{Results}

\section{IMT field mapping for in vitro DIPG cell treatment}

The in vitro IMT model utilized clinical grade, biocompatible, platinum (peripheral ground) and platinum-iridium (central stimulating) bioelectrodes fitted within $35 \mathrm{~mm}$ culture preparations of patient DIPG cells (Fig. 1a). A sinusoidal, biphasic waveform with peak-to-peak amplitude of $4 \mathrm{~V}$ was chosen to create reversing polarity and maximally disrupt the electrical environment using low intensity parameters known to be innocuous within the living brain (Fig. 1b) [11]. The $200 \mathrm{kHz}$ stimulation frequency is below that needed to produce thermal injury and surpassed the neuronal entrainment threshold to reduce the potential of off-target IMT effects when translated to eloquent brain regions [18-20]. The bioelectrode configuration and stimulation parameters created a symmetric, low intensity IMT field pattern across the DIPG cultures. The electric field was calculated by simulating the in vitro experiments in COMSOL Multiphysics (v 5.3a) using the electrode geometry presented and a constant voltage amplitude waveform generation. Based on this simulation, the largest electric field coverage extended concentrically from the central stimulating bioelectrode with smaller fields generated at regular intervals around the encircling peripheral bioelectrodes (Fig. 1c). The percent area coverage across the culture dish was calculated and plotted over one cycle of the waveform for electric field magnitude with thresholds in the range previously shown to be effective in other glioma cancers (Fig. 1d) [10, 11, 21, 22]. The coverage at the peak of the IMT waveform for each of the electric field magnitude thresholds of $1,0.75,0.5$ and $0.25 \mathrm{~V} / \mathrm{cm}$ was $6.2 \%, 8.9 \%, 16.4 \%$ and $54.7 \%$ respectively. Although other forms of glioma have been shown to require electric field amplitude $>1 \mathrm{~V} / \mathrm{cm}$ for optimal electrotherapy benefits, the threshold for DIPG response is not known [21, 22]. The therapeutic effects described below were therefore generated with this pilot IMT model providing electric field coverage $>0.25 \mathrm{~V} / \mathrm{cm}$ to roughly half, and $1 \mathrm{~V} / \mathrm{cm}$ to only a small fraction of the DIPG culture area.

\section{Patient DIPG cells are vulnerable to low intensity IMT fields}

Paired sham and IMT conditions were used to independently assess the impact of IMT monotherapy on primary DIPG cells (SU-DIPG-IV, SU-DIPG-XIX, SU-DIPG-XXIV) 

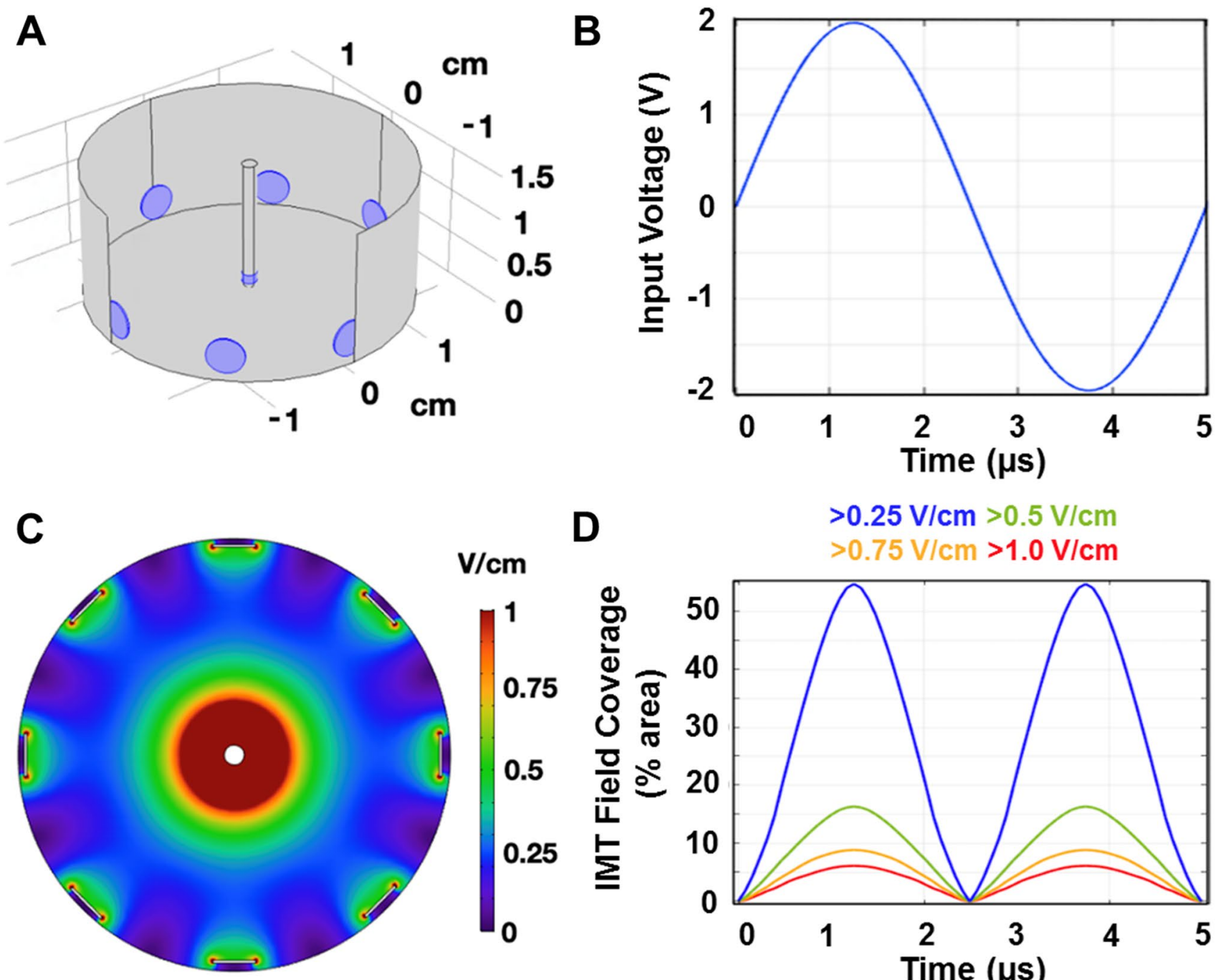

$\mathrm{V} / \mathrm{cm}$

D
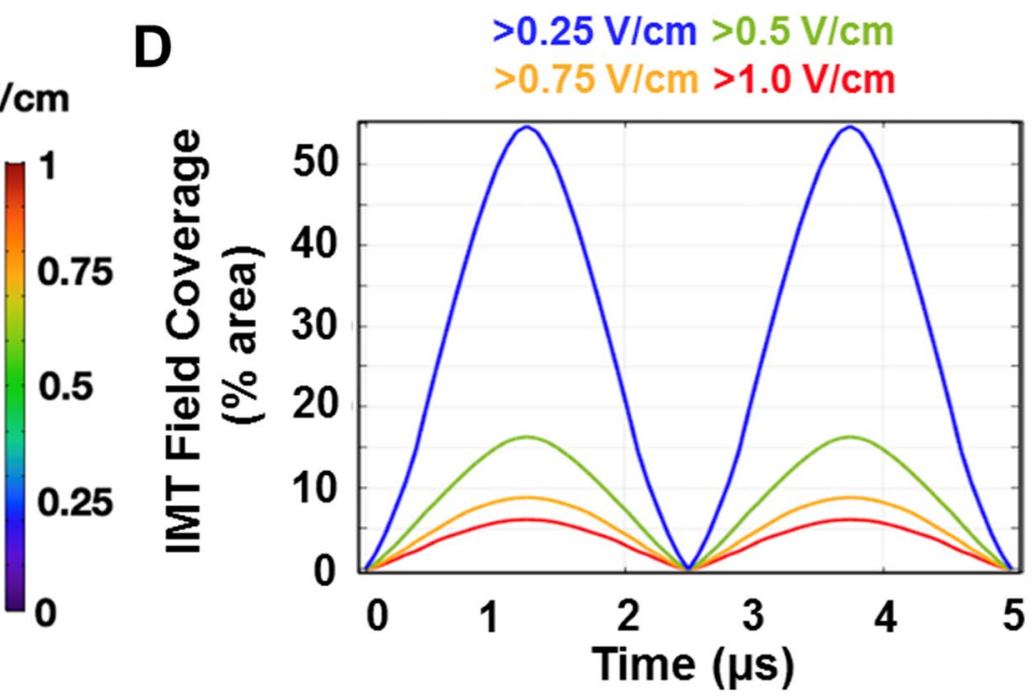

Fig. 1 Computer simulation of the IMT model. a The IMT model was created using a central stimulating bioelectrode placed in a $3.5 \mathrm{~cm}$ well along with eight peripheral grounded bioelectrodes. b A waveform generator supplied a $200 \mathrm{kHz}$ sinusoidal voltage with constant amplitude of $2 \mathrm{~V}$ to the central electrode. c Applying such parameters to this configuration of bioelectrodes resulted in an alter-

nating electric field with the predicted magnitude and distribution plotted as shown. $\mathbf{d}$ The percent area coverage across the culture dish reaching the electric field thresholds in the range anticipated to exert biological impact are plotted as a function of time over one cycle of the waveform. This pilot IMT system provided only fractional field coverage to the DIPG cultures

obtained from three pediatric patients. The 3-day exposure to low intensity IMT produced a dramatic and consistent reduction in tumor cell viability. IMT-treated cultures were sparse, pyknotic and exhibited faint MTT (formazan) labeling compared to sham-treated DIPG cultures (Fig. 2a, b). Statistical assessment performed on MTT measures normalized to those obtained in DIPG cells not exposed to IMT hardware (i.e., untreated controls) revealed a significant reduction in viability following IMT $(51.6 \pm 16.0 \%)$ compared to sham $(84.0 \pm 33.0 \%)$ treatment $(p=0.046$; Fig. $2 c)$. Flow cytometry was performed to confirm the MTT findings and evaluate the potential of apoptosis as a mechanism of IMT effect on DIPG cells. The ratio of viable:non-viable

DIPG cells decreased from $\sim 6: 1$ in sham-treated to $\sim 1.5: 1$ in IMT-treated conditions (Fig. 3). The significant reduction in DIPG cell viability was accompanied by a concordant rise in apoptotic and dead cell fractions across all patient samples (Table 1).

\section{IMT significantly enhances multi-modality treatment platforms for DIPG}

The impact of IMT monotherapy was compared to single agent TMZ chemotherapy and RT, as well as combined approaches using dual or triple modality platforms (Fig. 4). DIPG cells were treated with TMZ at a concentration 

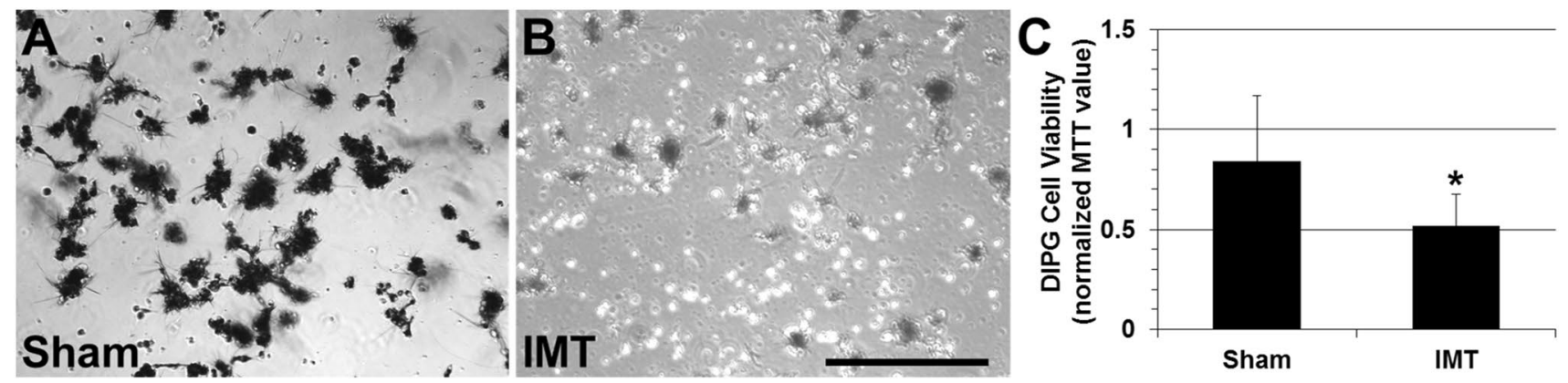

Fig. 2 DIPG cells are highly susceptible to low intensity IMT. Representative brightfield microscopy showing patient DIPG cultures following a 3-day exposure to a sham or b IMT conditions. The cultures were stained with the viability dye, MTT which produces a dark chromogen in viable cells. IMT-treated DIPG cells exhibited marked pyknosis with reduced MTT labeling and density. c MTT measures in sham and IMT treated cultures were normalized to those obtained in parallel cultures of untreated DIPG cells. The IMT exposure produced a marked and significant reduction in DIPG cells (asterisk; $\mathrm{p}=0.046 ; \mathrm{n}=3$; mean \pm standard deviation). Scale bar represents $500 \mu \mathrm{m}$ for $\mathbf{a}$ and $\mathbf{b}$
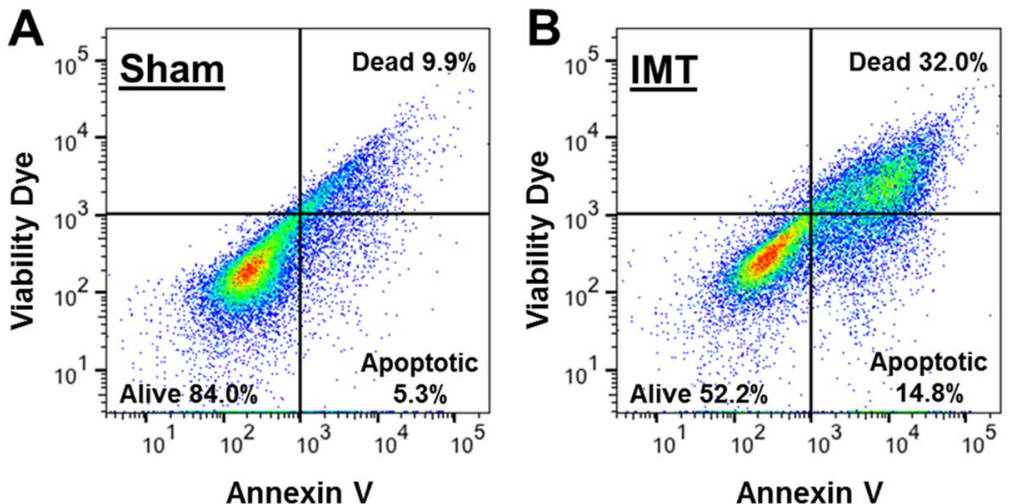

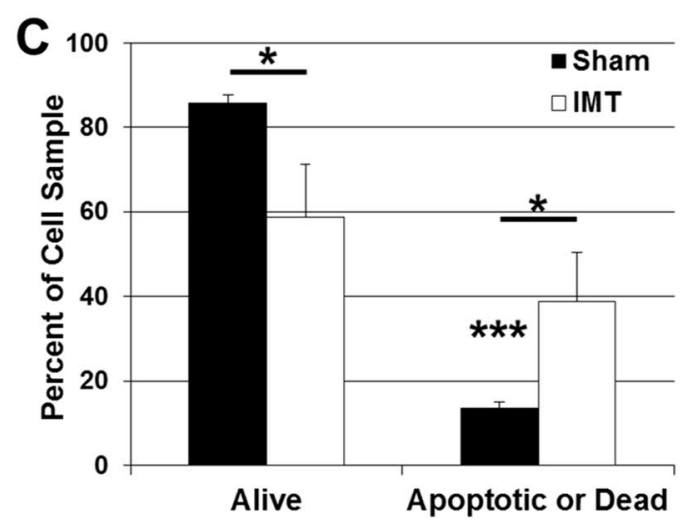

Fig. 3 IMT enhances apoptosis and death fractions in DIPG cell cultures. a Representative flow cytometry scatterplots of annexin and the viability dye zombie red (ZR) labeling of apoptotic and dead DIPG cells, respectively, after a 3-day exposure to a sham or b IMT conditions. c Quantification of live and combined apoptotic/dead DIPG cell fractions. In the sham-treated cultures, there was a marked, significant discrepancy between these fractions that was attenuated in IMT- treated cultures owing to the significant rise in cell death. Asterisks immediately above the histogram bars indicate significance between the live and apoptotic/dead fractions within the same treatment group. Significance between indicated data pairs is depicted by the asterisk above the horizontal bars. Data are presented as mean \pm standard deviation with significance indicated at $\mathrm{P}: *<0.05$ and $* * *<0.001$

Table 1 Summary of flow cytometry data in patient DIPG cell samples

\begin{tabular}{|c|c|c|c|c|c|c|c|c|c|c|}
\hline \multicolumn{2}{|c|}{ DIPG \# and cell fraction } & \multicolumn{3}{|c|}{ SU-DIPG-IV } & \multicolumn{3}{|c|}{ SU-DIPG-XIX } & \multicolumn{3}{|c|}{ SU-DIPG-XXIV } \\
\hline & & Live (\%) & Apoptotic (\%) & Dead (\%) & Live (\%) & Apoptotic (\%) & Dead $(\%)$ & Live (\%) & Apoptotic (\%) & Dead $(\%)$ \\
\hline \multirow[t]{2}{*}{ Treatment group } & Sham & 84 & 5.3 & 9.9 & 87.6 & 10 & 2.34 & 85.7 & 12.6 & 0.6 \\
\hline & IMT & 52.2 & 14.8 & 32 & 73 & 18.7 & 6.9 & 51.2 & 32.2 & 11.7 \\
\hline
\end{tabular}

equivalent to that achieved in plasma during standard care for GBM, another form of high grade glioma, with known modest, yet significant therapeutic efficacy and an acceptable toxicity profile [15]. The applied RT dose (4 Gy) in this study is known to have minimal impact in DIPG cells when used as a monotherapy, based on in-house experience (unpublished) and previous reports by other groups [16, 17].
This dose was chosen to determine if the negligible efficacy could be bolstered by the addition of TMZ and/or IMT to lessen the potential for adverse radiation effects when delivered in vivo. MTT analysis was used to assess the viability of DIPG cells following parallel 3-day exposures to sham IMT (i.e., hardware placement without stimulation) or IMT with or without TMZ, RT or combined TMZ-RT. All MTT 
measures were normalized to those of untreated DIPG controls. The sham IMT conditions had little effect on DIPG cells and yielded $96.0 \pm 6.6 \%$ viability. Monotherapy TMZ and RT was assessed in culture wells fitted with a sham IMT system. TMZ $(81.1 \pm 17 \%$ viability; $\mathrm{p}=0.564)$ and $\mathrm{RT}$ $(72.2 \pm 11.9 \%$ viability; $\mathrm{p}=0.105)$ produced slight reduction in MTT values that were not statistically different from the sham-only controls. In contrast, the combined TMZ-RT exposure (which also included the sham IMT hardware) produced a mean DIPG cell viability of $58.9 \pm 11.3 \%$ which was significantly reduced relative to the sham-only treated cells $(p=0.004)$. IMT monotherapy resulted in DIPG cell viability of $44.2 \pm 7.7 \%$ which was also significantly reduced compared to sham values $(\mathrm{p}<0.001)$ and significantly more effective than either TMZ $(p=0.005)$ or RT $(p=0.045)$ conditions. Dual therapy created by the addition of IMT significantly reduced DIPG cell viability compared to monotherapy TMZ $(81.1 \pm 17 \%$ vs. $40.1 \pm 2.50 \% ; \mathrm{p}=0.002)$ or RT $(72.2 \pm 11.9 \%$ vs. $34.7 \pm 4.4 ; \mathrm{p}=0.004)$. In contrast to TMZ and RT monotherapies, the MTT-measured impact of IMT alone was not significantly different than the combined TMZ-RT treatment $(p=0.614)$. Likewise, the therapeutic effect of combined TMZ-RT exposure was not significantly different from dual modality exposure to IMT-TMZ $(p=0.315)$ or IMT-RT $(p=0.113)$. Most notably, however, the impact of dual therapy TMZ-RT was potently enhanced by the addition of IMT in the multi-modality treatment platform TMZ-RT-IMT $(58.9 \pm 11.3 \%$ vs. $20.50 \pm 7.8 \%$ viability, respectively; $\mathrm{p}=0.004)$.

\section{Discussion}

This study provided novel evidence of DIPG cell vulnerability to low intensity electric fields delivered using an established preclinical IMT protocol. IMT produced apoptotic tumor cell death using stimulation parameters previously demonstrated to be non-injurious to normal neural cells in vitro and in vivo $[10,11]$. The applied stimulation frequency of $200 \mathrm{kHz}$ is above the range for neural entrainment and below that for thermal injury [19, 20]. IMT monotherapy reduced DIPG cell viability by $\sim 50 \%$ and was more efficacious than either low dose RT or a clinically utilized concentration of TMZ. The addition of IMT to the combined TMZ-RT paradigm dramatically reduced DIPG cell viability from $\sim 60 \%$ to $20 \%$. It remains unclear how IMT incites glioma cell death. There is evidence that non-ablative, non-thermal, low intensity electric fields work through multiple anti-neoplastic mechanisms that disrupt polarized molecules necessary for cell division, membrane permeability and channel homeostasis [23, 24]. In GBM studies, IMT has been shown to enhance the impact of TMZ and oncogene-targeted therapy as well as the uptake of hydrophilic genetic inhibitors [10]. The apparent lack of effect on postmitotic neurons or in normal brain parenchyma suggests that IMT exploits electrochemical vulnerabilities related to the neoplastic phenotype. Further studies are required to better understand these issues.

One possible method of providing IMT to DIPG entails custom configured, implanted bioelectrodes to perpetually deliver therapeutic electric fields across tumor-affected CNS regions using a concealed, titratable system that works cooperatively within a multi-modality treatment platform. Radiation is currently first line care for DIPG and, in this study, produced cooperative anti-neoplastic impact when delivered concurrently with IMT [4]. While chemotherapy has not shown significant benefit in DIPG for a potential host of reasons, TMZ is commonly used and beneficial in other forms of malignant glioma $[6,15]$. In this study, the insignificant impact of RT or TMZ monotherapies was in contradistinction to the potent effect of the triple IMT/RT/ TMZ combination, suggestive of sensitizing interactions that dramatically improve overall treatment efficacy. It remains to be determined if this efficacy will persist in vivo, and whether the multi-modality platform brings any unexpected toxicities. With respect to safety, a key putative advantage of the envisioned clinical IMT system is the ability to titrate, re-configure and discontinue stimulation. Reversibility of effect with therapy cessation, as typical with conventional forms of neuromodulation such as deep brain stimulation (DBS), is likely given the low amplitude and apparently benign nature of applied IMT fields in normal brain [11, 30-32].

The most readily available IMT prototype will likely include multi-contact leads stereotactically positioned within the brainstem and powered by an indwelling pulse generator. Surgical access to the brainstem is routinely achieved for various neoplastic and non-neoplastic indications and does not present an obvious barrier to development and safe application of IMT for DIPG patients. For example, there has been recent progress directly targeting DIPG tumors with catheters used for convection-enhanced delivery (CED) of pharmacotherapies $[25,26]$. While the outcome of the neoplastic disease was not altered using CED, there were no significant adversities that resulted from implanting catheters within the DIPG tumors. A longstanding, global experience with DBS provides another important example of the feasibility to implant hardware in the brainstem for treating neurological disease. DBS is the standard of surgical care for Parkinson's Disease (PD) where electrodes are chronically implanted into the subthalamic nucleus and often extending into the mesencephalic substantia nigra $[27,28]$. The pedunculopontine nucleus is another brainstem target being evaluated for implanted neuromodulation systems to improve postural and gait instability in PD [29]. It is important to note that DBS and IMT are starkly different 


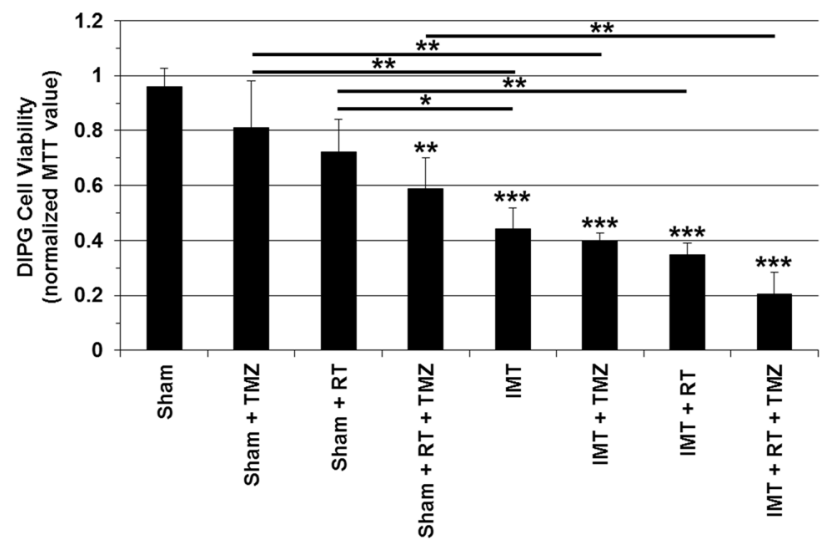

Fig. 4 IMT significantly augments multi-modality treatment platforms for DIPG. TMZ, low dose RT and IMT were applied to patient DIPG cultures in 3-day mono- and multi-modality therapies. The MTT viability assay was used to evaluate the impact of each treatment and the resultant data normalized to the mean MTT value obtained in untreated control DIPG cells. Monotherapy TMZ and RT were delivered in culture wells fitted with sham IMT hardware and neither had a significant impact on DIPG cells. In contrast, dual TMZ-RT exposure dramatically reduced DIPG cell viability. The IMT effect did not differ significantly from that of dual TMZ-RT therapy. However, the addition of IMT to the TMZ-RT combination produced marked and highly significant added therapeutic impact and a resultant DIPG viability of approximately $20 \%$ of untreated controls. Asterisks immediately above the histogram bars indicate significance between that specific treatment group and sham control. Significance between indicated data pairs is depicted by the asterisk(s) above the horizontal bars. Data are presented as mean \pm standard deviation with significance indicated at $\mathrm{P}$ : $*<0.05$, $* *<0.01$ and $* * *<0.001$

in neurological indication, operational parameters and hardware design. The electrical output of putative IMT systems will be defined by customized waveform, polarity, and stimulation parameters titrated to individual tumor response and regional anatomy. DBS technology typically delivers continuous, monophasic, square wave pulses at low frequency (e.g., 90-185 Hz) to disrupt and entrain pathological firing patterns [30-32]. In contrast, the present IMT system used an intermediate frequency $(200 \mathrm{kHz})$, sinusoidal waveform with reversing polarity intended to maximally disrupt electrical homeostasis in DIPG cells. The pulse frequency was 1000-fold higher than typical DBS settings and well out of range for neuronal entrainment in order to selectively target neoplastic cells while averting adverse neurological effects when stimulating tumor-infiltrated CNS regions $[11,18$, 33]. Additionally, the hardware configuration required to deliver personalized, comprehensive IMT field coverage will likely be sharply divergent from that of contemporary DBS electrodes designed to provide highly discrete zones of stimulation.

This proof-of-concept study provided novel evidence of DIPG cell susceptibility to low intensity electric fields delivered using an IMT strategy. The significant treatment response was produced in a pilot IMT model with incomplete field coverage, suggesting even greater efficacy may be realized using a comprehensive delivery system. Additionally, these DIPG cells were derived from fresh autopsy tissue that had been previously exposed to chemotherapy and RT, possibly selecting for tumor cells with heightened treatment resistance. The impact of IMT-based platforms measured in this study may therefore underestimate that attainable in treatment-naïve DIPG cells. While the present observations have yet to be replicated in larger, genetically-diverse cohorts and translational models, these exciting early data provide a new glimpse at the potential of electrotherapeutics to improve the otherwise devastating outcome for DIPG patients.

Acknowledgements We thank Dr. Michelle Monje (Stanford University) for kindly providing the human DIPG cells used in this study.

Funding This work was supported by the Cancer Research Society (S.S., E.W., M.O.H.), London Regional Cancer Program (M.O.H.), Western Innovation Fund (M.O.H) and Schulich School of Medicine and Dentistry (S.S., M.O.H.).

\section{Compliance with ethical standards}

Conflict of interest Matthew O. Hebb and Susanne Schmid are inventors on the following patent applications that are assigned to "London Health Sciences Centre Research Inc." (the applicant): PCT/ CA2016/050556 entitled "Intratumoral Modulation Therapy" and corresponding national phase applications in Canada, United States, Europe and China. The authors have no other competing interests.

Ethical approval All human DIPG cells were obtained from Dr. Michelle Monje (Stanford University) in accordance with the ethical standards of the Institutional Review Board (IRB) at Stanford University and with the 1964 Helsinki declaration and its later amendments or comparable ethical standards.

Informed consent All human tissues were obtained with informed consent for research purposes.

Open Access This article is distributed under the terms of the Creative Commons Attribution 4.0 International License (http://creativeco mmons.org/licenses/by/4.0/), which permits unrestricted use, distribution, and reproduction in any medium, provided you give appropriate credit to the original author(s) and the source, provide a link to the Creative Commons license, and indicate if changes were made.

\section{References}

1. Wu G, Broniscer A, McEachron TA, Lu C, Paugh BS, Becksfort J, Qu C, Ding L, Huether R, Parker M, Zhang J, Gajjar A, Dyer MA, Mullighan CG, Gilbertson RJ, Mardis ER, Wilson RK, Downing JR, Ellison DW, Zhang J, Baker SJ, St. Jude Children's Research Hospital-Washington University Pediatric Cancer Genome Project (2012) Somatic histone H3 alterations in pediatric diffuse 
intrinsic pontine gliomas and non-brainstem glioblastomas. Nat Genet 44:251-253

2. Johung TB, Monje M (2017) Diffuse intrinsic pontine glioma: new pathophysiological insights and emerging therapeutic targets. Curr Neuropharmacol 15(1):88-97

3. Harward S, Harrison Farber S, Malinzak M, Becher O, Thompson EM (2018) T2-weighted images are superior to other MR image types for the determination of diffuse intrinsic pontine glioma intratumoral heterogeneity. Childs Nerv Syst 34(3):449-455

4. Cohen KJ, Jabado N, Grill J (2017) Diffuse intrinsic pontine gliomas-current management and new biologic insights. Is there a glimmer of hope? Neuro oncology 19(8):1025-1034

5. Long W, Yi Y, Chen S, Cao Q, Zhao W, Liu Q (2017) Potential new therapies for pediatric diffuse intrinsic pontine glioma. Front Pharmacol 8:1-13

6. Gwak HS, Park HJ (2017) Developing chemotherapy for diffuse pontine intrinsic gliomas (DIPG). Crit Rev Oncol Hematol 120:111-119

7. Stupp R, Wong ET, Kanner AA, Steinberg D, Engelhard H, Heidecke V, Kirson ED, Taillibert S, Liebermann F, Dbalý V, Ram Z, Villano JL, Rainov N, Weinberg U, Schiff D, Kunschner L, Raizer J, Honnorat J, Sloan A, Malkin M, Landolfi JC, Payer F, Mehdorn M, Weil RJ, Pannullo SC, Westphal M, Smrcka M, Chin L, Kostron H, Hofer S, Bruce J, Cosgrove R, Paleologous N, Palti Y, Gutin PH (2012) NovoTTF-100A versus physician's choice chemotherapy in recurrent glioblastoma: a randomised phase III trial of a novel treatment modality. Eur J Cancer 48:2192-2202

8. Fonkem E, Wong ET (2012) NovoTTF-100A: a new treatment modality for recurrent glioblastoma. Expert Rev Neurother 12:895-899

9. Stupp R, Taillibert S, Kanner A, Read W, Steinberg D, Lhermitte B, Toms S, Idbaih A, Ahluwalia MS, Fink K, Di Meco F, Lieberman F, Zhu JJ, Stragliotto G, Tran D, Brem S, Hottinger A, Kirson ED, Lavy-Shahaf G, Weinberg U, Kim CY, Paek SH, Nicholas G, Bruna J, Hirte H, Weller M, Palti Y, Hegi ME, Ram Z (2017) Effect of tumor-treating fields plus maintenance temozolomide vs maintenance temozolomide alone on survival in patients with glioblastoma: a randomized clinical trial. JAMA 318:2306-2316

10. Xu H, Bihari F, Whitehead S, Wong E, Schmid S, Hebb MO (2016) In vitro validation of intratumoral modulation therapy for glioblastoma. Anticancer Res 36:71-80

11. Di Sebastiano AR, Deweyert A, Benoit S, Iredale E, Xu H, De Oliveira C, Wong E, Schmid S, Hebb MO (2018) Preclinical outcomes of intratumoral modulation therapy for glioblastoma. Sci Rep 8:7301

12. Lin GL, Monje M (2017) A protocol for rapid post-mortem cell culture of diffuse intrinsic pontine glioma (DIPG). J Vis Exp 121:e55360

13. Venkatesh HS, Tam LT, Woo PJ, Lennon J, Nagaraja S, Gillespie SM, Ni J, Duveau DY, Morris PJ, Zhao JJ, Thomas CJ, Monje M (2017) Targeting neuronal activity-regulated neuroligin-3 dependency in high-grade glioma. Nature 549(7673):533-537

14. Qin EY, Cooper DD, Abbott KL, Lennon J, Nagaraja S, Mackay A, Jones C, Vogel H, Jackson PK, Monje M (2017) Neural precursor-derived pleiotrophin mediates subventricular zone invasion by glioma. Cell 170(5):845-859

15. Ostermann S, Csajka C, Buclin T, Leyvraz S, Lejeune F, Decosterd LA, Stupp R (2004) Plasma and cerebrospinal fluid population pharmacokinetics of temozolomide in malignant glioma patients. Clin Cancer Res 10:3728-3736

16. Buch K, Peters T, Nawroth T, Sänger M, Schmidberger H, Langguth P (2012) Determination of cell survival after irradiation via clonogenic assay versus multiple MTT assay-a comparative study. Radiat Oncol 7:1
17. Smyth LM, Rogers PAW, Crosbie JC, Donoghue JF (2018) Characterization of diffuse intrinsic pontine glioma radiosensitivity using synchrotron microbeam radiotherapy and conventional radiation therapy in vitro. Radiat Res 189:146-155

18. Kirson ED, Gurvich Z, Schneiderman R, Dekel E, Itzhaki A, Wasserman Y, Schatzberger R, Palti Y (2004) Disruption of cancer cell replication by alternating electric fields. Cancer Res 64:3288-3295

19. Palti Y (1996) Stimulation of internal organs by means of external applied electrodes. J Appl Physiol 21:1619-1623

20. Storm FK, Morton DL, Kaiser LR, Harrison WH, Elliott RS, Weisenburger TH, Parker RG, Haskell CM (1982) Clinical radiofrequency hyperthermia: a review. Natl Cancer Inst Monogr 61:343-350

21. Wenger C, Salvador R, Basser PJ, Miranda PC (2015) The electric field distribution in the brain during TTFields therapy and its dependence on tissue dielectric properties and anatomy: a computational study. Phys Med Biol 60:7339-7357

22. Wenger C, Salvador R, Basser PJ, Miranda PC (2016) Improving tumor treating fields treatment efficacy in patients with glioblastoma using personalized array layouts. Int J Radiat Oncol Biol Phys 95:1137-1143

23. Pawłowski P, Szutowicz I, Marszałek P, Fikus M (1993) Bioelectrorheological model of the cell 5. Electrodestruction of cellular membrane in alternating electric field. Biophys J 65:541-549

24. Gera N, Yang A, Holtzman TS, Lee SX, Wong ET, Swanson KD (2015) Tumor treating fields perturb the localization of septins and cause aberrant mitotic exit. PLoS ONE 10:e0125269

25. Morgenstern PF, Zhou Z, Wembacher-Schröder E, Cina V, Tsiouris AJ, Souweidane MM (2018) Clinical tolerance of corticospinal tracts in convection-enhanced delivery to the brainstem. J Neurosurg 21:1-7

26. van Vuurden DG (2018) Convection-enhanced delivery: chemosurgery in diffuse intrinsic pontine glioma. Lancet Oncol 19(8):1001-1003

27. Habets JGV, Heijmans M, Kuijf ML, Janssen MLF, Temel Y, Kubben PL (2018) An update on adaptive deep brain stimulation in Parkinson's disease. Mov Disord 33(12):1834-1843

28. Ramirez-Zamora A, Ostrem JL (2018) Globus pallidus interna or subthalamic nucleus deep brain stimulation for Parkinson disease: a review. JAMA Neurol 75(3):367-372

29. Nowacki A, Galati S, Ai-Schlaeppi J, Bassetti C, Kaelin A, Pollo C (2018) Pedunculopontine nucleus: an integrative view with implications on deep brain stimulation. Neurobiol Dis. https:// doi.org/10.1016/j.nbd.2018.08.015

30. Udupa K, Chen R (2015) The mechanisms of action of deep brain stimulation and ideas for the future development. Prog Neurobiol 133:27-49

31. Wichmann T, DeLong M (2016) Deep brain stimulation for movement disorders of basal ganglia origin: restoring function or functionality? Neurotherapeutics 12:264-283

32. Herrington T, Cheng J, Eskandar E (2016) Mechanisms of deep brain stimulation. J Neurophysiol 115:19-38

33. Hottinger AF, Pacheco P, Stupp R (2016) Tumor treating fields: a novel treatment modality and its use in brain tumors. Neuro oncology 18:1338-1349

Publisher's Note Springer Nature remains neutral with regard to jurisdictional claims in published maps and institutional affiliations. 